\title{
Effect of seminal redox status on lipid peroxidation, apoptosis and DNA fragmentation in spermatozoa of infertile Saudi males
}

Sabiha Fatima, PhD, Raheek Alwaznah, MS, Ghadeer S. Aljuraiban, PhD, Samina Wasi, PhD,

Manal Abudawood, PhD, Mahmoud Abulmeaty, MD, Mohamed Y. Berika, PhD, Feda S. Aljaser, PhD.

\begin{abstract}

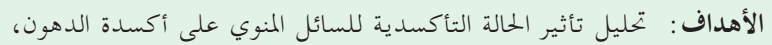

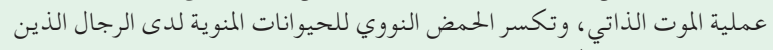
يعانون من ضعف الخصصوبة.

المنهجية: في هذه الدراسة المقطعية، تم تحليل حالة مضادات الأكسدة الكلية (TAS)

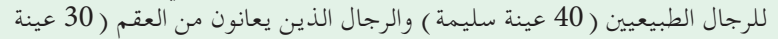

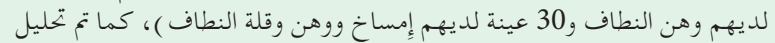

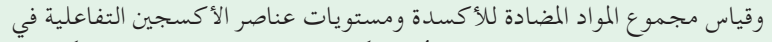

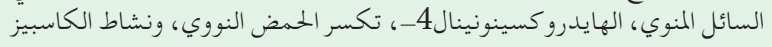
.3-

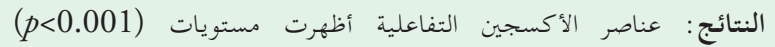

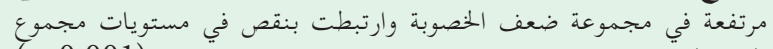

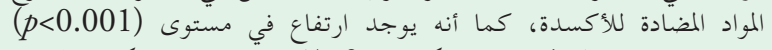

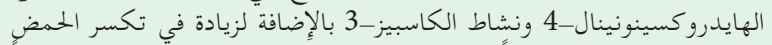

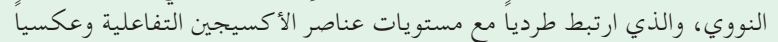

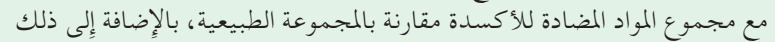

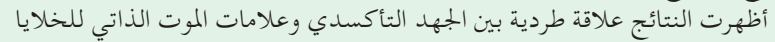
مع مؤشرات الحيوانات المنوية . معردية

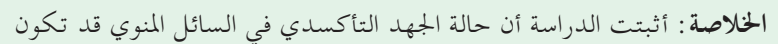

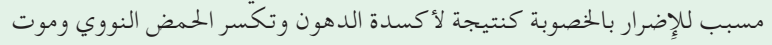

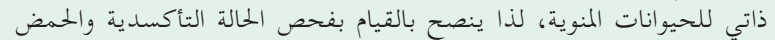

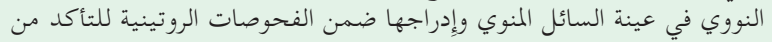

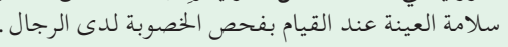

Objectives: To assess the effect of seminal redox status on lipid peroxidation (LPO), apoptosis and integrity of sperm DNA in infertile males.

Methods: In this case-control study, the total antioxidant status (TAS) and reactive oxygen species (ROS) levels were analyzed within the seminal plasma of fertile normozoospermic, $\mathrm{n}=40$ and infertile (asthenozoospermic, $\mathrm{n}=30$; oligoasthenoteratozoospermic, $\quad \mathrm{n}=30$ ) males. Additionally, the level of 4-hydroxynonenal (4-HNE), DNA fragmentation, and caspase-3 activity were estimated in the spermatozoa.
Results: Significantly $(p<0.001)$ increased seminal ROS level with decreased TAS scores was observed in the infertile groups compared to normozoospermics. The infertile males showed marked elevated $(p<0.001)$ levels of 4-HNE, DNA fragmentation and caspase-3 activity compared to normozoospermics, which was positively correlated to increased seminal ROS levels and negatively to the TAS score in the studied groups. Seminal ROS level was significantly inverse correlated to the semen parameters. Additionally, a strong negative correlation between DNA fragmentation, LPO, caspase-3activity and seminal parameters were observed.

Conclusion: Seminal oxidative stress is a potential risk factor for LPO, DNA damage, and apoptosis in spermatozoa, which can affect semen quality and male fertility. Thus, in addition to conventional seminological parameters, measurement of seminal oxidative stress and sperm DNA integrity may also be employed to investigate the functional integrity of spermatozoa at the molecular level.

Keywords: oxidative stress, lipid peroxidation, apoptosis, DNA fragmentation

Saudi Med J 2020; Vol. 41 (3): 238-246 doi: $10.15537 /$ smj.2020.3.24975

From the Department of Clinical Laboratory Sciences (Fatima, Alwaznah, Abudawood), from the Department of Community Health Sciences (Aljuraiban, Abulmeaty), from the Department of Rehabilitation Sciences (Berika); from the Chair of Medical and Molecular Genetics Research, Department of Clinical Laboratory Sciences (Aljaser), College of Applied Medical Sciences, King Saud University, Riyadh; and from the College of Medicine (Wasi), Imam Abdulrahman Bin Faisal University, Dammam, Kingdom of Saudi Arabia.

Received 11th November 2019. Accepted 3rd February 2020.

Address correspondence and reprint request to: Dr. Sabiha Fatima, Department of Clinical Laboratory Sciences, College of Applied Medical Sciences, King Saud University, Riyadh, Kingdom of Saudi Arabia. E-mail: sabmehdi@ksu.edu.sa

ORCID ID: https://orcid.org/0000-0001-8641-1369 
G lobally, approximately $15 \%$ of reproductive aged I couples have the problem of infertility. ${ }^{1,2}$ Male infertility has been found to be a major contributing factor to the overall infertility cases in Saudi Arabia., A one year retrospective study conducted on infertile couples reported a prevalence of approximately 19\% male infertility cases in the eastern province region of Saudi Arabia. ${ }^{5}$ According to the World Health Organization (WHO), semen quality is a key indicator of male reproductive health and fertility. ${ }^{6,7}$ Another recent retrospective study conducted in the western region of Saudi Arabia suggested the prevalence of abnormal semen parameters as the most common cause of subfertility in males. ${ }^{8}$ Abnormal semen characteristics can be caused by a variety of factors, including low sperm production and/or poor sperm quality. Currently, oxidative stress (OS) has been identified as a potential cause of idiopathic male infertility, which has detrimental effect on semen quality and fertility. ${ }^{10,11}$ Oxidative stress is the disturbance in the balance within the generation of the oxidants and the ability of antioxidant defenses to neutralize their harmful effects. The production of ROS is a normal cellular event in the male germline, and physiological ROS levels are required for sperm capacitation and hyperactivation; however, high ROS levels can affect normal spermatogenesis, and, consequently, sperm functions essential for successful fertilization. ${ }^{12,13}$

Sperm are rich in mitochondria, and most cellular reactive oxygen metabolites are generated through electron leakage from these organelles. ${ }^{14,15}$ Because they have a small volume of cytoplasm, sperm have reduced amounts of enzymatic antioxidants such as catalase, superoxide dismutase, and glutathione peroxidase, which limits their antioxidant defense capacity and renders them vulnerable to ROS attacks. ${ }^{16}$ As a consequence, spermatozoa are dependent on seminal plasma antioxidant defense mechanisms, which compensate for the deficiency in cytoplasmic enzymes. ${ }^{13,17}$ Nevertheless, sperm contain an abundance of substrates, such as polyunsaturated fatty acids (PUFAs) and DNA, which free radicals can attack. ${ }^{18}$ For instance, docosahexaenoic acid, one of the main PUFAs, comprises approximately $50 \%$ of the total content of spermatozoa and plays an

Disclosure. Authors have no conflict of interests, and the work was not supported or funded by any drug company. This study is funded by the Deanship of Scientific Research, King Saud University, Riyadh, Kingdom of Saudi Arabia (Research Group no. 1439-78). important function in controlling spermatogenesis, membrane flexibility and fluidity. ${ }^{19}$ Peroxidation of PUFAs generates lipid peroxyl radicals that can subsequently be converted into secondary aldehyde species like 4-hydroxynonenal (4-HNE), resulting in cellular damage. ${ }^{20}$ Oxidative stress can also disrupt sperm DNA integrity, thereby inducing a cellular, biochemical and morphological modifications that lead to apoptotic cell death. Caspase-3, a final executioner caspase, is recognized to play a pivotal role in apoptosis in different cell types, including spermatozoa. ${ }^{21}$ Thus, oxidative damage to the cell components may attribute to sperm defects and subfertility in males.

Although, infertility is a public health issue in the Saudi Arabia, showing a total conception rate drop from 7.3 to $3.03 \%$ during the period of 1970 to $2010,{ }^{4}$ the effect of seminal OS on the cellular damage and the functional integrity of spermatozoa influencing male infertility among the Saudi population has been a neglected area. Therefore, based on previous studies, the purpose of the our study was to assess and correlate the effect of seminal OS on sperm lipid peroxidation (LPO), DNA integrity, apoptosis, and semen variables in idiopathic infertile Saudi males in relation to fertile normozoospermic males as control.

Methods. All the reagents and chemicals used in our study were of analytical grade and were obtained from Sigma-Aldrich (St. Louis, Missouri, USA). The present case-control study was carried out in Assisted Conception Unit, King Khalid University Hospital, Riyadh, Saudi Arabia between December 2014 and 2015 after getting the approval from the Medical Ethics Committee, King Khalid University Hospital, Riyadh, Saudi Arabia. The study was conducted in accordance with the principles governing the Declaration of Helsinki. Semen samples were collected from 60 consenting male patients (25-50 years old). Forty fertile males with normal semen parameters were enrolled as controls. Complete medical history including reproductive history and evaluation of fertility was obtained from all the participants. Inclusion criteria includes infertile male participants in partner relationships with regular, unprotected coitus for at least 12 months without conception. Exclusion criteria for infertile males includes patients with any history of a prolonged or chronic condition such as diabetes, drug intake, smoking, systemic disease, testicular torsion, genitourinary anomaly, epididymal or vas deferens alteration, varicocele, inguinal surgery, vitamin intake, or antioxidant supplement treatment and the cases with female factor infertility were excluded from the study. Exclusion criteria also included azoospermia, 
leukocytospermia, incomplete semen analysis results, or semen samples inadequate for experimental parameter assessment. Semen collection and analysis was carried out by collecting ejaculates in sterile, non-toxic polypropylene containers (Alpha Laboratories, Eastleigh, UK) after an abstinence period of 2-3 days, according to the WHO guidelines (WHO 2010).22 The semen samples were placed in an incubator at $37^{\circ} \mathrm{C}$ for $30 \mathrm{~min}$ to approximately one hour for liquefaction. After complete liquefaction, semen volume and sperm parameters such as concentration, motility, and morphology were examined using a computer-assisted semen analysis (CASA) system (Hamilton Thorne Biosciences, Beverly, MA, USA), as per the WHO guidelines (WHO 2010). For each semen parameter analyzed, a $5-\mu \mathrm{L}$ aliquot was loaded into a counting chamber fitted with a heated stage set to $37^{\circ} \mathrm{C}$, and 4-10 fields were examined to ensure the accuracy of the CASA results; parallel manual assessment was also performed. Based on seminal characteristics, the participants were categorized into the following 3 groups: 1) Normozoospermics (control, $n=40$ ): subjects presenting with normal values for all semen parameters; sperm concentration of $\geq 15 \times 10^{6} \mathrm{sperm} / \mathrm{mL}$, total sperm motility $\geq 40 \%$, normal morphology in $\geq 4 \%$ of sperm. 2) Asthenozoospermics (AST, $n=30$ ): patients presenting with a normal sperm concentration of $\geq 15 \times$ $10^{6} \mathrm{sperm} / \mathrm{mL}$, a lower than normal level of total sperm motility $(<40 \%)$, and normal morphology in $\geq 4 \%$ of sperm and 3) Oligoasthenoteratozoospermics (OAT, $\mathrm{n}=30$ ): patients presenting with a lower than normal sperm concentration of $<15 \times 10^{6}$ sperm $/ \mathrm{mL}$, a lower than normal level for total sperm motility $(<40 \%)$, and normal morphology in $<4 \%$ of sperm.

After completing the semen analysis, the samples were divided into 2 parts. One aliquot was used to detect sperm DNA fragmentation by terminal deoxynucleotidyl transferase-mediated dUTP nick-end labeling (TUNEL) assay; the other aliquot was centrifuged for $15 \mathrm{~min}$ at $400 \times \mathrm{g}$. The supernatant containing the seminal plasma was collected and stored at $-80^{\circ} \mathrm{C}$ for assessment of ROS concentrations and total antioxidant status (TAS). The pellet containing the sperm cells at a concentration of $20 \times 10^{6} \mathrm{sperm} / \mathrm{mL}$ were washed twice with ice cold phosphate-buffered saline (PBS, pH 7.4), and then resuspended in $50 \mu \mathrm{L}$ of chilled cell lysis buffer.

Next, the cell lysates were centrifuged at $13000 \times \mathrm{g}$ for $10 \mathrm{~min}$ at $4^{\circ} \mathrm{C}$ to remove residual cell debris and the plasma-containing supernatant obtained was collected and stored at $-80^{\circ} \mathrm{C}$ for subsequent detection of $4-\mathrm{HNE}$ level and caspase-3 activity. The TAS, which provides information on the overall status of antioxidants in a biological sample, was measured using a colorimetric assay kit (EMD Millipore, MA, USA) following the instructions given by the manufacturer. The level of ROS production in seminal plasma was determined by OxiSelect ${ }^{\mathrm{TM}}$ (in vitro ROS/RNS Assay Kit from Cell Biolabs Inc., San Diego, CA, USA). The fluorescence intensity of $2^{\prime}, 7^{\prime}$-dichlorodihydrofluorescein (DCF) generated during the reaction was estimated at $480 \mathrm{~nm}$ excitation/530 nm emission wavelengths, which is proportional to ROS levels in the sample. OxiSelect ${ }^{\mathrm{TM}}$ 4-HNE-Adduct competitive ELISA Kit procured from Cell Biolabs Inc., San Diego, CA, USA was used to determine the concentration of 4-HNE, a biomarker of LPO in sperm cell lysates, following the instructions of the manufacturer. The amount of HNE adduct was determined at absorbance $450 \mathrm{~nm}$ using the HNE-BSA standard curve. Apoptotic cell number was detected by TUNEL assay using the FragEL ${ }^{\mathrm{TM}}$ DNA Fragmentation Detection Kit purchased from EMD Millipore, MA, USA. This method measures the endonuclease cleavage products obtained by enzymatically end-labeling DNA strand breaks. The semen aliquots were gently washed in PBS ( $\mathrm{pH} 7.4)$ by centrifugation $(1000 \mathrm{rpm})$ at $4^{\circ} \mathrm{C}$ for $5 \mathrm{~min}$. The pellet containing the cells were then fixed in $80 \%$ ethanol for at least $30 \mathrm{~min}$ at $4^{\circ} \mathrm{C}$, smeared onto slides, and then stored at $-20^{\circ} \mathrm{C}$ for TUNEL assay conducted as per the manufacturer's instructions. Sperm cells stained blue and green were observed under a fluorescence microscope (Zeiss, Oberkochen, Germany). The total sperm population was determined under blue fluorescence 4',6-diamidino-2-phenylindole (DAPI filter, ex 330-380) revealed the nuclei of living or dead cells. Sperm with intense green fluorescence (fluorescein filter, ex 465-495) represent TUNEL-positive cells with DNA strand breaks in their nuclei. Approximately 400 cells were counted on each slide, and the percentage of spermatozoa with fragmented DNA was calculated as the ratio of the total number of spermatozoa (green fluorescence) to the total number of counted cells (blue fluorescence) multiplied by 100 . Caspase 3 activity was measured using a Caspase-3 Colorimetric Assay kit (Abcam, Cambridge, UK) according to the manufacturer's instructions. The activity of caspase 3 was quantified spectrophotometrically at $405 \mathrm{~nm}$.

Statistical analysis. The mean \pm SD was calculated for each quantitative value. To assess the differences between the study groups were performed One-Way Analyses of Variance, followed by post hoc test, the Tukey's test and parametric paired t-test for the 
comparison between the 2 groups. The correlation between seminal redox status, oxidative biomarkers, DNA fragmentation levels, and sperm parameters were assessed by Spearman correlation analysis. In all cases, statistical significance was considered at $\mathrm{p}$-value of $<0.05$. The statistical analysis of all the data was conducted using IBM SPSS Statistics for Windows, Version 19.0 (IBM Corp., Armonk, N.Y., USA).

Results. Seminal parameter analysis. Seminal parameter analysis of the study groups showed insignificant differences in semen volume and mean age among the AST, OAT, and the normozoospermic control groups (Table 1). However, compared with normozoospermic group, AST and OAT had significantly lower $(p<0.05)$ sperm concentration, total motility and normal morphology. Table 1 summarizes the results of semen parameters within the study groups.

Total antioxidant status and ROS level in seminal plasma. Comparison of the ROS level in the seminal plasma of different study groups is shown in Figure 1A. The mean seminal ROS levels were significantly elevated in the AST and OAT $(p<0.001)$ groups compared to the normozoospermic. Figure $1 \mathrm{~B}$ shows the TAS score in the semen of the infertile groups compared to normozoospermic. The TAS was markedly lower in the AST $(p<0.001)$ and the OAT $(p<0.001)$ groups compared with the control group.

Lipid peroxidation products in the spermatozod. The level of 4-HNE, an electrophilic aldehyde and a natural by product of LPO compared with the level in

Table 1 - Analysis of conventional semen parameters in fertile normozoospermic (control) and infertile groups.

\begin{tabular}{|c|c|c|c|c|c|}
\hline Parameters & $\begin{array}{c}\text { Control } \\
(\mathrm{n}=30)\end{array}$ & $\begin{array}{c}\text { AST } \\
(n=40)\end{array}$ & $\begin{array}{c}\text { OAT } \\
(\mathrm{n}=30)\end{array}$ & $\begin{array}{c}P \text {-value } \\
\text { controls vs AST }\end{array}$ & $\begin{array}{l}\text { Controls vs } \\
\text { OAT }\end{array}$ \\
\hline Age & $37.50 \pm 4.91$ & $36.65 \pm 4.27$ & $38.74 \pm 5.41$ & $\begin{array}{l}0.163 \\
\text { NS }\end{array}$ & $\begin{array}{l}0.170 \\
\text { NS }\end{array}$ \\
\hline Volume (mL) & $3.38 \pm 2.17$ & $3.31 \pm 2.24$ & $3.32 \pm 2.22$ & $\begin{array}{l}0.465 \\
\text { NS }\end{array}$ & $\begin{array}{l}0.473 \\
\text { NS }\end{array}$ \\
\hline Sperm motility (\%) & $58.46 \pm 7.72$ & $30.12 \pm 5.46^{\dagger}$ & $24.16 \pm 5.11^{* *}$ & $<0.001$ & 0.001 \\
\hline Sperm concentration $\left(1 \times 10^{6} / \mathrm{mL}\right)$ & $78.41 \pm 6.83$ & $48.38 \pm 5.27^{\dagger}$ & $12.33 \pm 3.80^{* *}$ & $<0.001$ & $<0.001$ \\
\hline Normal sperm forms (\%) & $48.34 \pm 4.67$ & $33.25 \pm 4.13^{*}$ & $3.16 \pm 1.56^{* *}$ & 0.03 & $<0.001$ \\
\hline
\end{tabular}

Values represent means \pm SD. NS: non-significant, AST: asthenozoospermic, OAT: oligoasthenoteratozoospermic, ${ }^{*} p \leq 0.05,{ }^{\dagger} p \leq 0.01$
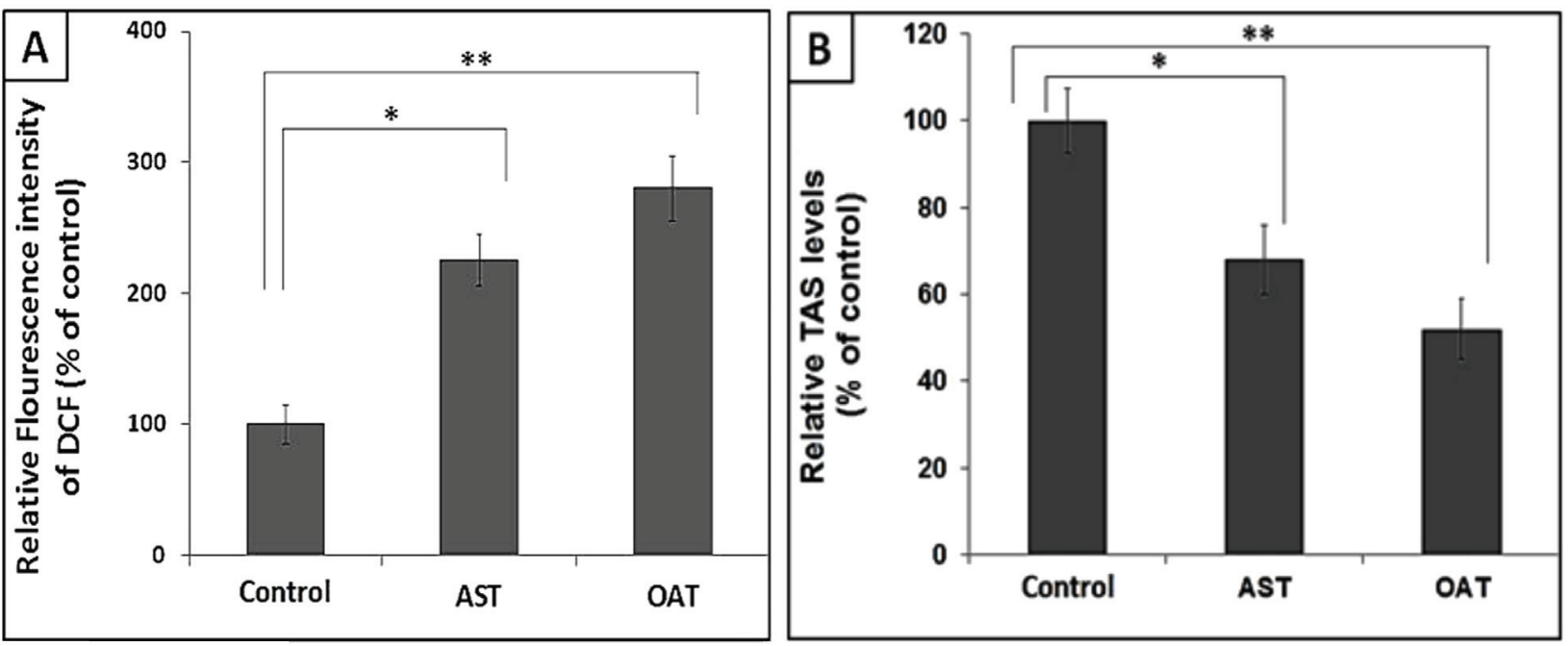

Figure 1 - Comparison of seminal A) reactive oxygen species (ROS) levels and B) total antioxidant status (TAS) between the fertile normozoospermic (control) and asthenozoospermic (AST) and oligoasthenoteratozoospermic (OAT) groups. The level of ROS was significantly higher whereas, TAS score in both infertile groups was significantly lower compared to normozoospermic group. Values represent means \pm SD. ${ }^{*} p<0.001$, AST group versus the control group; ${ }^{* *} p<0.001$, OAT group versus the control group. 
the fertile normozoospermic group manifested a highly significant increase in both the OAT $(p<0.001)$ and AST ( $p=0.003)$ groups (Figure 2).

Apoptosis and DNA fragmentation. Caspase 3, a final executor of apoptosis, was measured to determine the effect of seminal ROS generation on the apoptosis of spermatozoa (Figure 3). Compared to the values observed in the normozoospermic group, mean caspase- 3 activity of the OAT $(p<0.001)$ and AST $(p=0.005)$ groups showed significantly higher fold increase. As illustrated in Figure 4, the percentage of DNA fragmentation was significantly greater $(p<0.001)$ in the OAT group and the AST group compared to normozoospermic subjects.

Correlation between seminal redox status and sperm 4-HNE, DNA fragmentation, caspase-3 activity, and conventional semen parameters. Among all the studied groups a relationship between seminal TAS, seminal ROS, sperm oxidative stress markers, DNA fragmentation, and conventional semen parameters were analyzed (Table 2). In the seminal plasma, the increase in ROS level was negatively correlated $(\mathrm{r}=-0.51$, $p<0.001)$ with the TAS. Seminal ROS levels showed a strong positive correlation with 4-HNE levels $(\mathrm{r}=0.46$, $p<0.001)$, caspase 3 activity $(\mathrm{r}=0.53, p<0.001)$, and DNA fragmentation levels $(\mathrm{r}=0.48, p<0.001)$. The observed decrease in seminal TAS values in infertile males was significantly inverse correlated with the

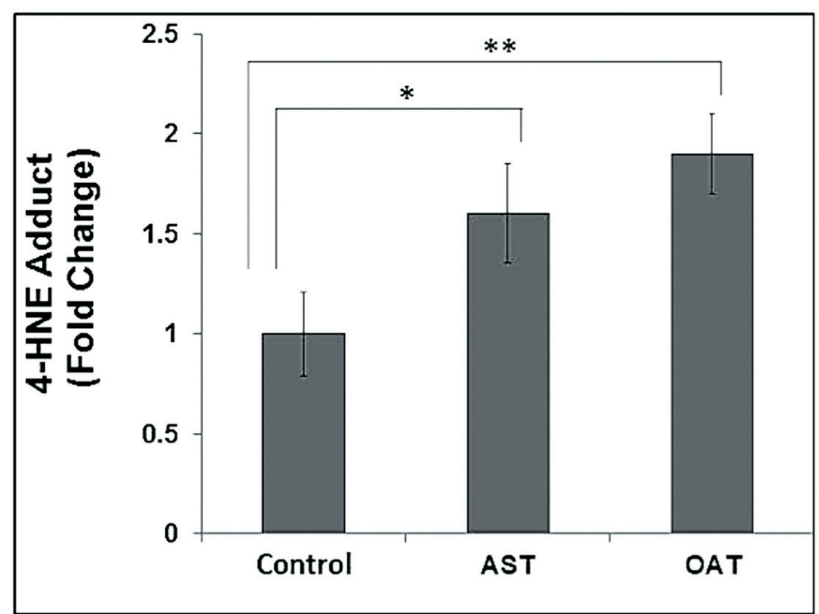

Figure 2 - Comparison of the levels of 4-hydroxynonenal (4-HNE) in sperm cell lysates derived from the fertile normozoospermic (control) and asthenozoospermic (AST) and oligoasthenoteratozoospermic (OAT) males. The mean 4-HNE level in both infertile groups was significantly higher than that in the control group. Values represent means \pm SD. ${ }^{*} p=0.003$, AST group vs the control group; ${ }^{* *} p<0.001$, OAT group versus the control group. increased caspase- 3 activity $(\mathrm{r}=-0.22, \quad p=0.028)$, $4 \mathrm{HNE}(\mathrm{r}=-0.36, p=0.002)$ and DNA fragmentation $(\mathrm{r}=-0.39, p<0.001)$ levels. Furthermore, seminal ROS levels showed a significantly strong negative correlation with total sperm concentration $(\mathrm{r}=-0.38, p=0.003)$. A similar strongly negative correlation was observed in the total sperm motility $(\mathrm{r}=-0.56, p<0.001)$, and normal sperm morphology $(\mathrm{r}=-0.61, p=0.001)$ among the study population. Increased DNA fragmentation and caspase-3 activity also showed significantly strong negative correlation with the decreased sperm motility $(\mathrm{r}=-0.58, p<0.001 ; \mathrm{r}=-0.51, p<0.001$, respectively) and normal sperm morphology $(\mathrm{r}=-0.48, p<0.001$; $\mathrm{r}=-0.56, \quad p<0.001)$ for all the studied groups. Moreover, decreased sperm concentration manifested no significant correlation with DNA fragmentation and caspase-3 activity. We also evaluated correlations between sperm 4-HNE levels and semen parameters. A significant negative correlation was observed between 4-HNE level, total sperm motility $(r=-0.62, p<0.001)$ and normal sperm morphology $(\mathrm{r}=-0.53, p<0.001)$. Sperm concentration also showed inverse association ( $\mathrm{r}=-0.37, p=0.004)$ with 4-HNE level among the study population. Further, sperm 4-HNE levels were positively associated with the percentage of DNA damage $(\mathrm{r}=0.52, p<0.001)$ and caspase-3 activity $(\mathrm{r}=0.48, p<0.001)$.

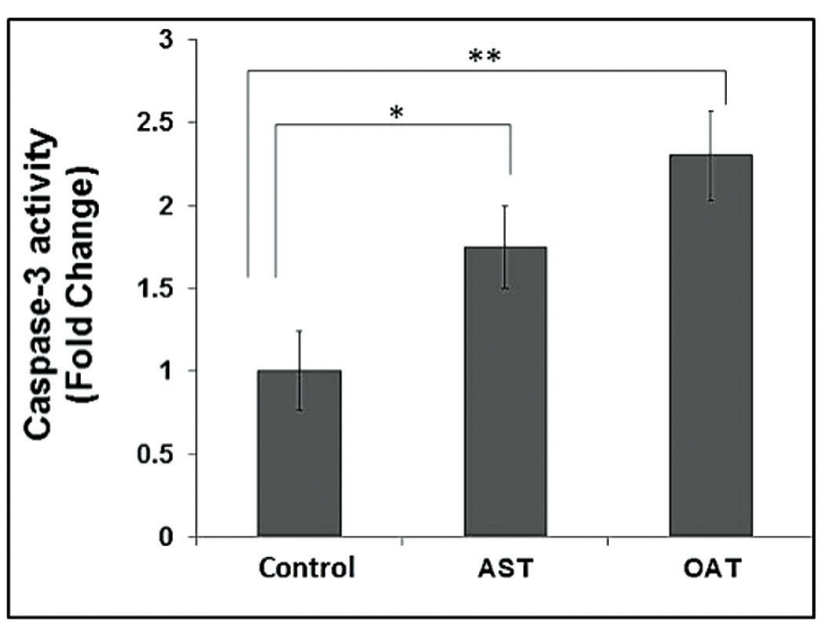

Figure 3 - Comparison of the caspase- 3 activity in sperm cell lysates derived from the fertile normozoospermic (control) and asthenozoospermic (AST) and oligoasthenoteratozoospermic (OAT) groups. The mean caspase- 3 activity in both infertile groups was significantly higher than that in the control group. Values represent means $\pm \mathrm{SD}$. ${ }^{*} \mathrm{p}<0.005$, AST group versus the control group; ${ }^{* *} p<0.001$, OAT group versus the control group. 


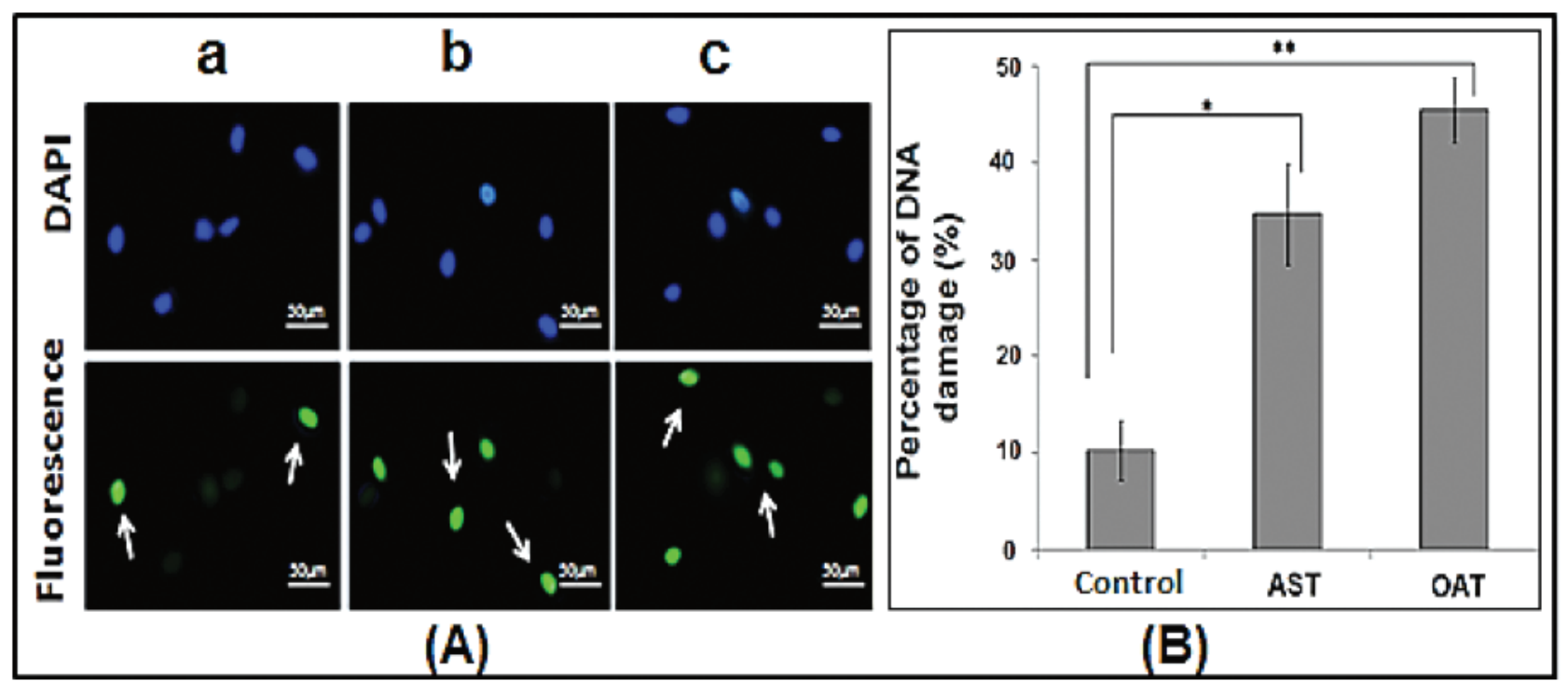

Figure 4 - DNA fragmentation in human sperm as determined by TUNEL assay. A) Fluorescence microscopy images of sperm derived from men in (a) the fertile normozoospermic group (control), (b) asthenozoospermic (AST) group, and (c) oligoasthenoteratozoospermic (OAT) group. White arrows indicate TUNEL-positive nuclei (bright green), while DAPI (blue) staining indicates the total number of nuclei (scale bar: $50 \mu \mathrm{m}$ ). B) Quantitative determination (percentage of apoptotic cells versus the total number of cells) of DNA fragmentation in sperm of males in the control, AST, and OAT groups. A greater number of TUNEL-positive nuclei were observed in the OAT and AST groups than in the control group. Data are expressed as means \pm SD. ${ }^{*} p<0.001$ control versus AST; ${ }^{* *} p<0.001$ control versus OAT.

Table 2 - Correlation between seminal redox status, the caspase-3 activity, levels of sperm hydroxynonenal, DNA fragmentation, and conventional semen parameters.

\begin{tabular}{|c|c|c|c|c|c|c|c|c|}
\hline Parameters & ROS & TAS & 4-HNE & $\begin{array}{c}\text { Caspase-3 } \\
\text { activity }\end{array}$ & $\begin{array}{c}\% \text { DNA } \\
\text { fragmentation }\end{array}$ & $\begin{array}{c}\text { Total } \\
\text { motility }\end{array}$ & $\begin{array}{c}\text { Sperm } \\
\text { concentration }\end{array}$ & $\begin{array}{c}\text { Normal sperm } \\
\text { morphology }\end{array}$ \\
\hline ROS & - & $\begin{array}{c}\mathrm{r}=-0.51^{* *} \\
p=0.001\end{array}$ & $\begin{array}{l}\mathrm{r}=0.53^{* *} \\
p<0.001\end{array}$ & $\begin{array}{l}r=0.46^{* *} \\
p<0.001\end{array}$ & $\begin{array}{l}r=0.48^{* *} \\
p<0.001\end{array}$ & $\begin{array}{c}\mathrm{r}=-0.56^{* *} \\
p<0.001\end{array}$ & $\begin{array}{l}\mathrm{r}=-0.38^{*} \\
p=0.006\end{array}$ & $\begin{array}{c}\mathrm{r}=-0.61^{* *} \\
p<0.001\end{array}$ \\
\hline TAS & - & - & $\begin{array}{l}\mathrm{r}=-0.36^{*} \\
p=0.002\end{array}$ & $\begin{array}{l}\mathrm{r}=-0.22^{*} \\
p=0.028\end{array}$ & $\begin{array}{c}\mathrm{r}=-0.39^{* *} \\
p=0.001\end{array}$ & $\begin{array}{l}\mathrm{r}=0.33^{*} \\
p=0.005\end{array}$ & $\begin{array}{l}\mathrm{r}=0.18^{*} \\
p=0.034\end{array}$ & $\begin{array}{l}\mathrm{r}=0.46^{* *} \\
p<0.001\end{array}$ \\
\hline 4-HNE & - & - & - & $\begin{array}{l}\mathrm{r}=0.48^{* *} \\
p<0.001\end{array}$ & $\begin{array}{l}\mathrm{r}=0.52^{* *} \\
p<0.001\end{array}$ & $\begin{array}{c}\mathrm{r}=-0.62^{* *} \\
p<0.001\end{array}$ & $\begin{array}{l}\mathrm{r}=-0.37^{*} \\
p=0.004\end{array}$ & $\begin{array}{c}\mathrm{r}=-0.44^{* *} \\
p<0.001\end{array}$ \\
\hline $\begin{array}{l}\text { Caspase-3 } \\
\text { activity }\end{array}$ & - & - & - & - & $\begin{array}{l}r=0.58^{* *} \\
p<0.001\end{array}$ & $\begin{array}{c}\mathrm{r}=-0.51^{* *} \\
p<0.001\end{array}$ & $\begin{array}{c}\mathrm{r}=-0.046 \\
p=0.32 \\
\mathrm{NS}\end{array}$ & $\begin{array}{c}\mathrm{r}=-0.56^{* *} \\
p<0.001\end{array}$ \\
\hline $\begin{array}{l}\text { DNA } \\
\text { fragmentation }\end{array}$ & - & - & - & - & - & $\begin{array}{c}\mathrm{r}=-0.58^{* *} \\
p<0.001\end{array}$ & $\begin{array}{c}\mathrm{r}=0.057 \\
p=0.51 \\
\mathrm{NS}\end{array}$ & $\begin{array}{c}\mathrm{r}=-0.54^{* *} \\
p<0.001\end{array}$ \\
\hline $\begin{array}{l}\text { Total } \\
\text { motility }\end{array}$ & - & - & - & - & - & - & $\begin{array}{c}r=0.058^{*} \\
p=0.007\end{array}$ & $\begin{array}{l}r=0.49^{* *} \\
p<0.001\end{array}$ \\
\hline $\begin{array}{l}\text { Sperm } \\
\text { concentration }\end{array}$ & - & - & - & - & - & - & - & $\begin{array}{l}\mathrm{r}=0.58^{* *} \\
p<0.001\end{array}$ \\
\hline
\end{tabular}

NS: non-significant, ROS: reactive oxygen species, TAS: total antioxidant status, 4-HNE: 4-hydroxynonenal, ${ }^{*} p \leq 0.05,{ }^{* *} p \leq 0.01$ 
Discussion. Overproduction of ROS and limited antioxidant defenses offered by spermatozoa render human sperm extremely susceptible to OS. ${ }^{23}$ In the present study, we found impaired oxidant scavenging capacity and high ROS levels in the seminal plasma of infertile Saudi males. These results agree with those of several studies that have implicated increased OS in seminal plasma as a major pathology associated with infertility in approximately $30-80 \%$ of the infertile men. ${ }^{17,24}$ High ROS levels can be an important risk factor for oxidant-induced damage to the components of the cell including proteins, DNA, and PUFAs. ${ }^{25}$ The unconjugated double bonds present adjoining a methylene group in PUFAs weakens methyl carbonhydrogen interactions; this makes hydrogen atoms more susceptible to abstraction and renders PUFAs vulnerable to oxidative damage. ${ }^{26}$ Spermatozoa depend on seminal antioxidant defense mechanisms owing to their limited antioxidant capacity. ${ }^{17}$ We found that the concentration of 4-HNE, a cytotoxic LPO byproduct, was higher in cell lysates of sperm from both infertile groups than in those of the fertile group. As observed in previous studies, our investigation showed a strong correlation between seminal ROS and 4-HNE levels in spermatozoa. ${ }^{27}$ 4-hydroxynonenal is one of the most physiologically active lipids, with the capacity to modify any cellular protein. ${ }^{28,29}$ Lipid peroxidation, which can lead to almost $60 \%$ loss of the fatty acid content of the sperm plasma membrane, can impair membrane fluidity and increase membrane ionic permeability. Electrophilic lipid aldehydes can form an adduct with the proteins that control sperm movement. ${ }^{30}$ Further, LPO affects the axonemal protein phosphorylation and inactivates membrane-bound receptors and enzymes, resulting in sperm immobilization that consequently alters sperm motility, morphology, and concentration. ${ }^{31}$ Also, in agreement with previous studies, we observed a strongly negative correlation of 4-HNE with sperm motility and normal morphology (Table 2).

Reactive oxygen species by oxidative modifications of the nitrogenous bases can directly cause damage to the sperm DNA. Moreover, deficient sperm protamination, observed in most infertile men, makes their sperm DNA more vulnerable to attack by ROS. ${ }^{32}$ Spermatozoa have limited DNA repair mechanism for ROS-induced damage. Extensive DNA damage induces DNA fragmentation, a biochemical hallmark of apoptosis, through activation of caspases. In this study, we detected higher levels of caspase 3, a final executor of apoptosis, in spermatozoa of infertile patients compared with those of fertile subjects (Figure 4). Although, some degree of apoptosis is always required for efficient delivery of healthy sperm, dysregulated apoptosis in mature spermatozoa is suggested to be involved in the induction of a subfertility state and is associated with increased DNA fragmentation. ${ }^{33,34}$ The percentage of DNA fragmentation was greater in the OAT and AST groups than in the fertile group (Figure 5). The increased seminal OS and sperm DNA fragmentation in the OAT group compared to AST group indicates, that the morphologically abnormal spermatozoa, with a greater volume of residual cytoplasm and reduced antioxidant defense, may be more vulnerable to DNA strand breaks. ${ }^{35}$

Regarding the association between seminal OS, apoptosis, and sperm parameters, we observed a significant positive correlation between caspase-3 activity and reduced sperm concentration, sperm motility, and the number of sperms showing normal morphology. This relationship is similar to that reported in other studies, which showed that increased apoptotic rates decrease sperm concentration and motility. ${ }^{34}$ The inverse correlated of Sperm DNA fragmentation with the total motility and normal morphology of spermatozoa, could be the reason of subfertility in the patient groups. ${ }^{36-38}$ Significantly positive correlation recorded between seminal OS, LPO and DNA fragmentation in our study suggests that the observed LPO and DNA damage may have been due to increased ROS production. The high susceptibility of spermatozoa to OS-induced DNA damage can result not only in poor fertilization rates but also lead to poor embryo quality which can cause birth defects and even childhood diseases. ${ }^{23,39}$ Abnormal sperm morphology associated with the retention of residual cytoplasm in OAT is known to promote the generation of pathogenic levels of endogenous ROS in spermatozoa. ${ }^{40}$ Consequently, the patients presenting with OAT showed higher levels of ROS, apoptosis, and sperm DNA fragmentation compared with those presenting with AST. One of the causes of the idiopathic male infertility among the Saudi population can be the hot climate of Saudi Arabia throughout most of the year, which may raise the intra-scrotal temperature and may lead to OS induced damage to sperm macromolecules, affecting sperm quality. ${ }^{8,41}$

In conclusion, we observed that a firm association exists between an imbalance in seminal redox status with increased sperm LPO, apoptosis, DNA damage, and abnormal conventional seminal parameters among infertile Saudi males. The higher the seminal ROS levels, 
the greater the LPO, apoptosis, and DNA damage observed in spermatozoa. This indicates, that imbalance in seminal redox status can cause oxidative modification of sperm macromolecules, which affects the sperm quality and function. The association of subcellular factors in infertility cannot be explained clearly with conventional semen analysis. Thus, data from the present study suggests that during the investigation of male infertility, along with the standardized assays, assessment of oxidative status and sperm DNA fragmentation is recommended. Although, our study has tried to analyzed the underlying cause of abnormal semen parameters in infertile Saudi males, the limitation to the study is the small sample size and also we did not analyzed the enzymatic and nonenzymatic antioxidants in seminal plasma of infertile male. Therefore, in future research, a large scale study must be conducted to explore the seminal concentration of enzymatic and nonenzymatic antioxidants and their repercussions on genetic predisposition and sperm quality among the infertile Saudi males.

Acknowledgment. Authors gratefully acknowledge the Deanship of Scientific Research at King Saud University, Riyadh, Kingdom of Saudi Arabia, for funding this research (Research Group no. 1439-78). We would like to thank "Research Support and Services Unit, King Saud University" for English language editing. We gratefully acknowledge Mrs. Hajera Tabassum for her assistance and contribution in experimental procedures.

\section{References}

1. Inhorn MC, Patrizio P. Infertility around the globe: new thinking on the gender, and reproductive technology and global movement in 21st centuary. Hum Reprod Update. 2015; 21 : 411-426.

2. Velu A, Prasad G. Epidemiologic aspects of male infertility. Int J Reprod Contracept Obstet Gynecol 2017; 6: 3362-3365.

3. AlEnezi H, Isa AM, Abu-Rafea B, Madbouly K, Binsaleh S. Pattern of semen fluid abnormalities in male partners of infertile couples in Riyadh, Saudi Arabia. Can J Urol. 2014; 21 : 7322-7325.

4. Binsaleh S, Al-Qahtani R, Madbouly K, Isa AM, Abu-Rafea B. Evaluation of sperm DNA damage in men from infertile Saudi couples. J Reprod Med. 2015; 60: 135-140.

5. Al-Turki HA. Prevalence of primary and secondary infertility from tertiary center in eastern Saudi Arabia. Middle East Fertil Soc J 2015; 20: 237-240.

6. Patel AS, Leong JY, Ramasamy R. Prediction of male infertility by the World Health Organization laboratory manual for assessment of semen analysis: A systematic review. Arab J Urol 2017; 16: 96-102.

7. Barratt CLR, Björndahl L, De Jonge CJ, Lamb DJ, Osorio Martini F, et al. The diagnosis of male infertility: an analysis of the evidence to support the development of global WHO guidance-challenges and future research opportunities. Hum Reprod Update 2017; 23: 660-680.
8. Alasmari W, Edris F, Albar Z, Gari A, Eskandar M, Al Fageah M, Zawawi S. High proportion of abnormal semen characteristics among saudi infertile couples. Clin Med Diagn. 2018; 8: 14-18.

9. Amjad S, Baig M, Zahid N, Tariq S, Rehman R. Association between leptin, obesity, hormonal interplay and male infertility. Andrologia 2019; 51: e13147.

10. Dorostghoal M, Kazeminejad SR, Shahbazian N, Pourmehdi M, Jabbari A. Oxidative stress status and sperm DNA fragmentation in fertile and infertile men. Andrologia 2017; 49: e12762.

11. Sun J, Wang H, Liu B, Shi W, Shi J, Zhang Z, Xing J. Rutin attenuates $\mathrm{H} 2 \mathrm{O} 2$-induced oxidation damage and apoptosis in Leydig cells by activating PI3K/Akt signal pathways. Biomed Pharmacother 2017; 88: 500-506.

12. Dutta S, Majzoub A, Agarwal A. Oxidative stress and sperm function: A systematic review on evaluation and management. Arab J Urol. 2019; 17: 87-97.

13. Sabeti P, Pourmasumi S, Rahiminia T, Akyash F, Talebi AR. Etiologies of sperm oxidative stress. Int J Reprod Biomed (Yazd). 2016; 14: 231-240.

14. Zorov DB, Juhaszova M, Sollott SJ. Mitochondrial reactive oxygen species (ROS) and ROS-induced ROS release. Physiol Rev 2014; 94: 909-950.

15. Santini SJ, Cordone V, Mijit M, Tatone C, Amicarelli F, Di Emidio G. Role of mitochondria in the oxidative stress induced by electromagnetic fields: focus on reproductive system. Oxid Med Cell Longev. 2018; 5076271.

16. Fatima $S$. Role of reactive oxygen species in male reproduction. In: Novel Prospects Based on Oxidative/Nitrosative Stress in Health and Disease. Croatia: INTECH publisher; 2018.

17. Huang C, Cao X, Pang D, Li C, Luo Q, Zou Y, et al. Is male infertility associated with increased oxidative stress in seminal plasma? A-meta analysis. Oncotarget 2018; 9: 24494-24513.

18. Guerriero G, Trocchia S, Abdel-Gawad FK, Ciarcia G. Roles of reactive oxygen species in the spermatogenesis regulation. Front Endocrinol (Lausanne) 2014; 5: 56.

19. Alahmar AT. Role of oxidative stress in male infertility: An updated review. J Hum Reprod. Sci. 2019; 12: 4-18.

20. Mustafa A, Alfaqih MA, Al-Shaboul O. The 4-hydroxynonenal mediated oxidative damage of blood proteins and lipids involves secondary lipid peroxidation reactions. Exp Ther Med. 2018; 16: 2132-2137.

21. Prokhorova EA, Kopeina GS, Lavrik IN, Zhivotovsky B. Apoptosis regulation by subcellular relocation of caspases. Sci Rep 2018; 8: 12199.

22. WHO 2010 Semen analysis: Standard procedure. In Examination and processing of human semen, edn 5, pp 10-114. Cambridge: Cambridg University Press.

23. Bisht S, Faiq M, Tolahunase M, Dada R. Oxidative stress and male infertility. Nat Rev Urol 2017; 14: 470-485.

24. Wagner H, Cheng JW, Ko EY. Role of reactive oxygen species in male infertility: An updated review of literature. Arab J Urol 2017; 16: 35-43.

25. Atig F, Kerkeni A, Saad A, Ajina M. Effects of reduced seminal enzymatic antioxidants on sperm DNA fragmentation and semen quality of Tunisian infertile men. J Assist Reprod Genet 2017; 34: 373-381.

26. Van Tran L, Malla BA, Kumar S, Tyagi AK. Polyunsaturated Fatty Acids in Male Ruminant Reproduction - A Review. AsianAustralas J Anim Sci 2017; 30: 622-637. 
27. Walters JLH, De Iuliis GN, Nixon B, Bromfield EG. Oxidative Stress in the Male Germline: A Review of Novel Strategies to Reduce 4-Hydroxynonenal Production. Antioxidants (Basel) 2018; 7: pii: E132.

28. Hall SE, Aitken RJ, Nixon B, Smith ND, Gibb Z. Electrophilic aldehyde products of lipid peroxidation selectively adduct to heat shock protein 90 and arylsulfatase $A$ in stallion spermatozoa. Biol Reprod. 2017; 96 : 107-121.

29. Baker MA, Weinberg A, Hetherington L, Villaverde AI, Velkov T, BaellJ, et al. Defining the mechanisms by which the reactive oxygen species by-product, 4-hydroxynonenal, affects human sperm cell function. Biol Reprod. 2015; 92: 108.

30. Barik G, Chaturvedula L, Bobby Z. Role of Oxidative Stress and Antioxidants in Male Infertility: An Interventional Study. $J$ Hum Reprod Sci 2019; 12: 204-209.

31. Agarwal A, Virk G, Ong C, du Plessis SS. Effect of oxidative stress on male reproduction. World J Mens Health 2014; 32: $1-17$.

32. Ni K, Spiess AN, Schuppe HC, Steger K. The impact of sperm protamine deficiency and sperm DNA damage on human male fertility: a systematic review and meta-analysis. Andrology 2016; 4: 789-99.

33. Engel KM, Springsguth CH, Grunewald S. What happens to the unsuccessful spermatozoa? Andrology 2018; 6: 335-344.

34. Nakidkina AN., Kuzmina TI. Apoptosis in Spermatozoa and Its Role in Deteriorating Semen Quality. Russ J Dev Biol 2019; 50: 165-172.
35. Sá R, Cunha M, Rocha E, Barros A, Sousa M. Sperm DNA fragmentation is related to sperm morphological staining patterns. Reprod Biomed Online 2015; 31: 506-515.

36. Aydos OS, Yükselten Y, Kaplan F, Sunguroğlu A, Aydos K. Analysis of the correlation between sperm DNA integrity and conventional semen parameters in infertile men. Turk J Urol 2015; 41: 191-197.

37. Binsaleh S, Al-Qahtani R, Madbouly K, Isa AM, Abu-Rafea B. Evaluation of sperm DNA damage in men from infertile Saudi couples. J Reprod Med 2015; 60: 135-140.

38. Al Omrani B, Al Eisa N, Javed M, Al Ghedan M, Al Matrafi $\mathrm{H}$, Al Sufyan H. Associations of sperm DNA fragmentation with lifestyle factors and semen parameters of Saudi men and its impact on ICSI outcome. Reprod Biol Endocrinol 2018; 16 : 49.

39. Agarwal A, Cho CL, Esteves SC, Majzoub A. Current limitation and future perspective of sperm DNA fragmentation tests. Transl Androl Urol 2017; 6: S549-S552.

40. Majzoub A, Agarwal A. Antioxidant therapy in idiopathic oligoasthenoteratozoospermia. Indian J Urol 2017; 33: 207-214.

41. Al-Otaibi ST. Male infertility among bakers associated with exposure to high environmental temperature at the work place. J Taibah Univ Med Sci 2018; 13: 103-107. 